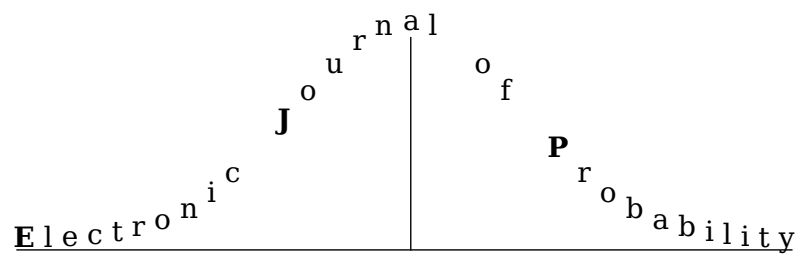

Electron. J. Probab. 26 (2021), article no. 98, 1-19.

ISSN: 1083-6489 https://doi.org/10.1214/21-EJP645

\title{
The speed of random walk on Galton-Watson trees with vanishing conductances
}

\author{
Tabea Glatzel ${ }^{*} \quad$ Jan Nagel ${ }^{\dagger}$
}

\begin{abstract}
In this paper we consider random walks on Galton-Watson trees with random conductances. On these trees, the distance of the walker to the root satisfies a law of large numbers with limit the effective velocity, or speed of the walk. We study the regularity of the speed as a function of the distribution of conductances, in particular when the distribution of conductances converges to a non-elliptic limit.
\end{abstract}

Keywords: random walk in random environment; Galton-Watson trees; effective velocity MSC2020 subject classifications: 60K37; 60F15; 60J80; 60K40.

Submitted to EJP on December 4, 2020, final version accepted on May 4, 2021.

\section{Introduction}

Let $T$ be a supercritical Galton-Watson tree and let $\left(X_{n}\right)_{n \geq 0}$ be a simple random walk on $T$, starting at the root. It is well known since the work of [23], that $\left(X_{n}\right)_{n \geq 0}$ moves away from the root with a linear rate,

$$
\lim _{n \rightarrow \infty} \frac{\left|X_{n}\right|}{n}=v \text { a.s., }
$$

where $\left|X_{n}\right|$ denotes the distance of $X_{n}$ from the root and the limit holds almost surely with respect to the annealed law, that is, averaged over the random walk and the tree, and conditioned on $T$ being infinite. The limit $v$ is a positive constant and is called the effective velocity of the random walk. In fact, $v$ can be given explicitly in terms of the offspring law. Such a law of large numbers still holds for a random walk on a GaltonWatson tree with random conductances. For this process, given the Galton-Watson tree $T$, the edges of the tree are assigned random positive conductances, independently and identically distributed for different edges and the random walk traverses an edge with a probability proportional to the conductance of that edge. It was proven by

\footnotetext{
${ }^{*}$ TU Dortmund, Fakultät für Mathematik, Vogelpothsweg 87, 44227 Dortmund, Germany. E-mail: tabea . glatzel@tu-dortmund.de

${ }^{\dagger}$ TU Dortmund, Fakultät für Mathematik, Vogelpothsweg 87, 44227 Dortmund, Germany. E-mail: jan. nagel@tu-dortmund.de
} 
The speed of random walk on Galton-Watson trees with vanishing conductances

[19], that a convergence as in (1.1) still holds, when we now also average over the conductances, with a limit depending on the offspring law of the tree and the law of the conductances. [19] also give a formula for the speed as an expectation of ratios of effective conductances. Unfortunately, this means that the speed cannot be explicitly computed even for very simple distributions of conductances.

In this paper, we study the regularity of the speed as a function of the distribution of the conductances. In particular, we investigate how the speed changes when the conductances on a positive fraction of edges go to zero. The regularity of the speed as a function of the local transition probabilities is a prominent question for random walk on Galton-Watson trees. In particular, it has been well studied for biased random walks, when the conductances of edges between the $n$-th and the $(n+1)$-th generation in the tree are multiplied by $\lambda^{-n}$, for a bias parameter $\lambda>0$, see [24]. A formula for the asymptotic velocity was proven by [1]. Similar to the formula of [19], the speed may be written as a function of effective conductances of the tree, but it also depends in a highly nontrivial way on $\lambda$. The bias can induce a strong trapping phenomenon, see for example [4,13,10] or [3] for an overview. The monotonicity of $v$ as a function of $\lambda$ has been studied by [5], the behavior for $\lambda$ close to the recurrent regime by [6] and differentiability by [11]. Closely related are results for random walks in $\mathbb{Z}^{d}$ with random conductances and bias parameter $\lambda$, as studied by [17, 18, 7], or for the Mott random walk $[15,16]$. The regularity of the speed on the tree as a function of the offspring law was studied in [20], when the offspring law is close to criticality.

Before we present our results, let us introduce the model more precisely. Let $\Omega$ be the set of all triples $(T, \rho, \xi)$, where $T$ is a tree with $\operatorname{root} \rho$ and $\mathcal{E}(T)$ its set of undirected edges and $\xi \in[0, \infty)^{\mathcal{E}(T)}$ is a configuration of conductances on the edges of $T$. An element $\omega \in \Omega$ is called an environment. We then let $\mathrm{P}$ be the law on $\Omega$, such that under $\mathrm{P}, T$ is a Galton-Watson tree with offspring law $\nu$ and $\operatorname{root} \rho$ and conditioned on $T$, the conductances $\xi$ are independent and identically distributed with marginal law $\mu$. Let $N$ be the number of offspring of the root $\rho$. We assume that the tree is supercritical, $\mathrm{E}[N]>1$, it has no leaves, $\mathrm{P}(N=0)=0$ and the second moment $E\left[N^{2}\right]$ is finite. It is also possible to consider Galton-Watson trees with leaves, i.e., $\mathrm{P}(N=0)>0$. In this case, one has to consider the various probability measures conditioned on non-extinction. For simplicity, we assume the tree to have no leaves. The conductances are assumed to be uniformly elliptic, that is, there exists a $\delta>0$, such that

$$
\mu\left(\left[\delta, \delta^{-1}\right]\right)=1 .
$$

For vertices $u, v \in T$, we write $u \sim v$, if $u$ and $v$ are connected by an edge in $T$ and we say that $u$ and $v$ are neighbors. We denote the graph distance of a vertex $v \in T$ to the root $\rho$ by $|v|$ and let $\operatorname{deg}(v)$ be the degree of vertex $v$.

Given an environment $\omega=(T, \rho, \xi)$, we let $P_{\omega}$ be the quenched law of the Markov chain $\left(X_{n}\right)_{n \geq 0}$ on $T$, starting at $\rho$ and with transition probabilities

$$
P_{\omega}\left(X_{n+1}=v \mid X_{n}=u\right)=\frac{\xi(u, v)}{\sum_{w \sim u} \xi(u, w)}
$$

if $u \sim v$. The annealed law $\mathbb{P}$ is then defined by

$$
\mathbb{P}(A \times B)=\int_{A} P_{\omega}(B) \mathrm{dP}(\omega)
$$

for measurable subsets $A \times B$ of the set of all pairs $\left(\omega, T^{\mathbb{N}_{0}}\right.$ ) (as usual, the $\sigma$-algebras are generated by finite-dimensional projections). We may identify a Galton-Watson tree as a subtree of the Ulam-Harris tree $\mathbb{T}$ (see e.g. [21]), so that $\mathbb{P}$ is a measure on $\Omega \times \mathbb{T}^{\mathbb{N}_{0}}$. 
The speed of random walk on Galton-Watson trees with vanishing conductances

As proven by [19], we then have

$$
\lim _{n \rightarrow \infty} \frac{\left|X_{n}\right|}{n}=v=v(\nu, \mu) \quad \mathbb{P}-\text { almost surely. }
$$

In Section 2, we recall a formula for $v$. We are interested in the behavior of $v(\nu, \mu)$ as a function of $\mu$, the marginal law of the conductances. As a first result, we have that $v$ is continuous, as long as we stay in the framework of uniformly elliptic laws. More precisely, to guarantee the convergence of $v\left(\nu, \mu_{n}\right)$ for a sequence of distributions of conductances $\mu_{n}$, we require the $\mu_{n}$ to be uniformly elliptic with the same ellipticity constant $\delta$.

Proposition 1.1. For any $\delta>0$, the mapping $\mu \mapsto v(\nu, \mu)$ is continuous on the set of uniformly elliptic measures satisfying (1.2), equipped with the weak topoplogy.

We want to investigate the speed when some of the conductances approach zero. Define for $\mu$ satisfying (1.2) for some $\delta>0$ and for $\varepsilon \geq 0, \alpha \in(0,1)$ the measure

$$
\mu_{\varepsilon}=\alpha \delta_{\varepsilon}+(1-\alpha) \mu,
$$

where $\delta_{\varepsilon}$ denotes the Dirac measure in $\varepsilon$. Note that for any $\varepsilon>0$, the measure $\mu_{\varepsilon}$ is still uniformly elliptic. There is a natural way of coupling weighted trees $(T, \xi)$ with marginal conductance law $\mu$ with trees with conductances distributed according to $\mu_{\varepsilon}$. For each edge in $T$ we toss an independent coin with success probability $\alpha$ to replace the current conductance by $\varepsilon$. For $\varepsilon=0$, the subtree $T_{0}$ formed by edges with positive conductances might be finite, and then the random walk can only move on a finite tree. In fact, if $\varepsilon=0$ and the pruned Galton-Watson process obtained by removing $\varepsilon$-edges is not supercritical, $T_{0}$ is finite with probability 1 and we set $v\left(\nu, \mu_{0}\right)=0$. If $\varepsilon=0$ and $T_{0}$ is supercritical, it is infinite with positive probability and then the speed is usually defined when we condition on $T_{0}$ to be infinite. In this case, and consistently with the definitions in [23] and [19], we define the conditioned environment law $\overline{\mathrm{P}}=\mathrm{P}\left(\cdot|| T_{0} \mid=\infty\right)$ and the conditioned annealed law $\overline{\mathrm{P}}$ as in (1.4), with $\mathrm{P}$ replaced by $\overline{\mathrm{P}}$. Then $v\left(\nu, \mu_{0}\right)$ is the almost sure limit of $\left|X_{n}\right| / n$ under $\overline{\mathbb{P}}$, when the (traversable) tree is conditioned to be infinite [19, Remark 4.1]. The following theorem gives the limit of the speed as $\varepsilon$ tends to zero.

Theorem 1.2. It holds that

$$
\lim _{\varepsilon \searrow 0} v\left(\nu, \mu_{\varepsilon}\right)=\hat{\mathrm{P}}_{0}\left(\left|T_{0}\right|=\infty\right) \cdot v\left(\nu, \mu_{0}\right)
$$

where $\hat{\mathrm{P}}_{0}$ is the invariant measure for the tree seen from the random walk (see Section 2).

Theorem 1.2 shows that the limit of the speed for conductances approaching zero is not the speed of the random walk as usually defined on trees with positive extinction probability. Instead it is smaller by the multiplicative constant $\hat{\mathrm{P}}_{0}\left(\left|T_{0}\right|=\infty\right)<1$. The reason for this is a slowdown experienced by the walk as it spends time in finite parts of the tree which can only be left by traversing an edge with conductance $\varepsilon$, acting as traps in the environment. Indeed, for small $\varepsilon$, the tree can be thought of as a forest formed by the edges with conductance larger than $\varepsilon$, connected by $\varepsilon$-edges. The walker can only enter or leave the finite trees formed by large conductances by moving along an $\varepsilon$-edge, which has a probability of order $\varepsilon$. Consequently, the time spent on finite trees connected by large conductances is of order $\varepsilon^{-1}$, resulting in a macroscopic amount of time spend in traps. For $\varepsilon=0$ and conditioned on $T_{0}$ being infinite, as in the definition of $v\left(\nu, \mu_{0}\right)$, such a slowdown cannot occur. It is interesting to note that when $T_{0}$ is conditioned to be infinite, the random walk moves on a tree with leaves, which create a different slowdown 


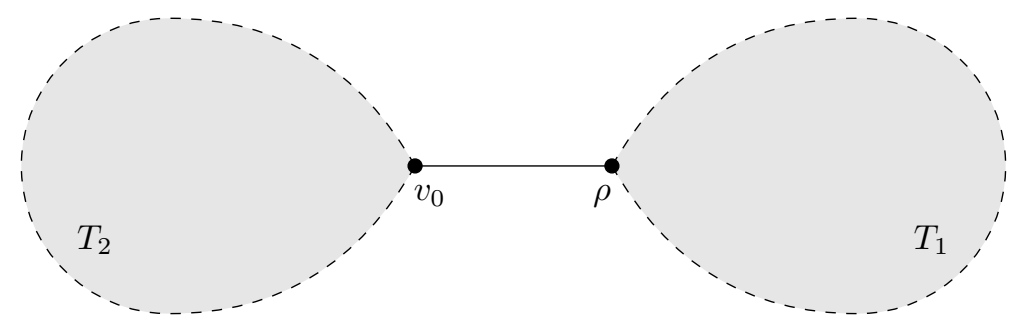

Figure 1: Under $\mathrm{P}_{\varepsilon}^{\text {aug }}$ the subtrees $T_{1}$ and $T_{2}$ are independent and identically distributed weighted Galton-Watson trees with law $\mathrm{P}_{\varepsilon}$.

mechanism by forming traps. The even smaller limit of $v\left(\nu, \mu_{\varepsilon}\right)$ shows that the latter effect is in some sense weaker.

The slowdown is not just given by the probability for an infinite tree under $\mathrm{P}$, since the random walk can only move with linear speed when we see an infinite tree from the perspective of the walk after a long time. We therefore have to look for an infinite tree under the measure $\hat{\mathrm{P}}_{0}$, which generates a tree stationary for the process of the environment viewed from the random walk.

Our arguments rely heavily on the assumption of uniform ellipticity. In fact, when $\mu((0, \theta))>0$ for all $\theta>0$, another trapping phenomenon arises, where the random walk may spend a long time on an $\varepsilon$-edge surrounded by edges with very small conductance. We suspect that in this case, a convergence as in Theorem 1.2 may depend on the tail of $\mu$ at 0 .

In the next section, we introduce the invariant measure for the environment seen from the walker and its limit as $\varepsilon \rightarrow 0$. The proofs of Proposition 1.1 and Theorem 1.2 are given in Section 3.

\section{The invariant measure}

In order to define the invariant measure, we need to introduce augmented GaltonWatson trees and bi-infinite walks on them. We follow [19], with modified notation. We write $\mathrm{P}_{\varepsilon}$ for $\mathrm{P}$, if the marginal law of the conductances is given by $\mu_{\varepsilon}$ as in (1.6). Then, let $\mathrm{P}_{\varepsilon}^{\text {aug }}$ be the law on $\Omega$, such that under $\mathrm{P}_{\varepsilon}^{\text {aug }}, T$ is an augmented Galton-Watson tree with an additional edge $\left(\rho, v_{0}\right)$ added to the root, and attached to vertex $v_{0}$ at the other end of that edge an independent Galton-Watson tree, see Figure 1. That is,

$$
\mathrm{P}_{\varepsilon}^{\operatorname{aug}}((T, \rho, \xi) \in A)=\sum_{k=1}^{\infty} p_{k-1} \mathrm{P}_{\varepsilon}((T, \rho, \xi) \in A \mid \operatorname{deg}(\rho)=k) .
$$

The measure $\hat{\mathrm{P}}_{\varepsilon}$ is then defined via

$$
\hat{\mathrm{E}}_{\varepsilon}[f(T, \rho, \xi)]=\mathrm{E}_{\varepsilon}^{\mathrm{aug}}\left[f(T, \rho, \xi) \frac{\pi(\rho)}{\gamma \operatorname{deg}(\rho)}\right],
$$

with $\pi(v)=\sum_{w \sim v} \xi(v, w)$ and $\gamma$ the normalization constant. Under $\hat{\mathrm{P}}_{\varepsilon}$, the root is weighted by the average conductance of adjacent edges and $\gamma$ is the mean conductance of an edge. Given an environment $\omega \in \Omega$, let $\hat{P}_{\omega}$ be the law of the bi-infinite random walk $\left(X_{n}\right)_{n \in \mathbb{Z}}$ on $T$, such that $X_{0}=\rho$ and $\left(X_{n}\right)_{n \geq 0}$ (interpreted as the future of the random walk) and $\left(X_{-n}\right)_{n \geq 0}$ (interpreted as the past of the random walk) are independent with marginal law as defined by (1.3). We let $\hat{\mathbb{P}}_{\varepsilon}$ be the corresponding annealed law, defined analogously to (1.4) on $\Omega \times \mathbb{T}^{\mathbb{Z}}$. As verified in [19, Equation (2.2)], we have for any 
The speed of random walk on Galton-Watson trees with vanishing conductances

bounded and measureable functions $f, g$

$$
\hat{\mathrm{E}}_{\varepsilon}\left[f(T, \rho, \xi) g\left(T, v_{0}, \xi\right) \xi\left(\rho, v_{0}\right)\right]=\hat{\mathrm{E}}_{\varepsilon}\left[f\left(T, v_{0}, \xi\right) g(T, \rho, \xi) \xi\left(v_{0}, \rho\right)\right] .
$$

The environment seen from the random walk is the process $\left(T, X_{n}, \xi\right)_{n \in \mathbb{Z}}$ with state space $\Omega$ and transition operator

$$
G f(T, \rho, \xi)=\frac{1}{\pi(\rho)} \sum_{v \sim \rho} \xi(\rho, v) f(T, v, \xi) .
$$

Using the symmetry relation (2.3), it is then proven in [19, Lemma 3.1] that $G$ is reversible with respect to $\hat{\mathrm{P}}_{\varepsilon}$,

$$
\hat{\mathrm{E}}_{\varepsilon}\left[f(T, \rho, \xi) G g\left(T, v_{0}, \xi\right)\right]=\hat{\mathrm{E}}_{\varepsilon}\left[G f(T, \rho, \xi) g\left(T, v_{0}, \xi\right)\right]
$$

for $f, g \in L^{2}\left(\hat{\mathrm{P}}_{\varepsilon}\right)$. In particular, $\hat{\mathrm{P}}_{\varepsilon}$ is an invariant measure for the environment seen from the random walk and then the sequence $\left(T, X_{n}, \xi\right)_{n}$ of the tree seen from the random walk is stationary under $\hat{\mathbb{P}}_{\varepsilon}$. In the reference, the invariance is proven for uniformly elliptic conductances, but it holds for $\varepsilon=0$ as well.

In order to prove Theorem 1.2, we need to consider $\hat{\mathrm{P}}_{\varepsilon}$ as $\varepsilon \rightarrow 0$. Note that all the measures introduced in this section are well defined also if $\varepsilon=0$, i.e., when some edges have conductance 0 . We then have the following continuity in $\varepsilon$, the proof is in Section 3.

Lemma 2.1. As $\varepsilon \rightarrow 0$, we have $\hat{\mathrm{P}}_{\varepsilon} \rightarrow \hat{\mathrm{P}}_{0}$ and $\hat{\mathrm{P}}_{\varepsilon} \rightarrow \hat{\mathbb{P}}_{0}$ weakly.

Remark 2.2. Let us comment on the limit measure $\hat{\mathrm{P}}_{0}$. If $\varepsilon=0$, the edges with positive conductance form a forest of disconnected trees. Since the random walk can only traverse edges with positive conductance, it moves on a subgraph of the original tree $T$. The measure $\hat{\mathrm{P}}_{0}$ is still invariant for the environment seen from the random walk. It is however not the unique invariant measure anymore. For example, any measure under which $\{\pi(\rho)=0\}$ has probability 1 is trivially invariant. Since the measure $\hat{\mathrm{P}}_{0}$ is the weak limit of $\hat{\mathrm{P}}_{\varepsilon}$, it is the right measure to consider for the limit of the speed. Note that the event $\{\pi(\rho)=0\}$ has probability 0 under $\hat{\mathrm{P}}_{0}$ as defined in (2.2), so there is always at least one edge with positive conductance adjacent to $\rho$. Indeed, for $\varepsilon>0$ small the vertices $v$ with $\pi(v)=\varepsilon \operatorname{deg}(v)$ can only be reached by an $\varepsilon$-edge, which happens rarely, but they don't trap the random walk: the next step from $v$ is chosen uniformly among its neighbors. Therefore, the fraction of time spent in such vertices vanishes as $\varepsilon \rightarrow 0$.

For vertices $u, v, z \in T$, we define the signed distance of $u$ to $v$ with respect to $z$ as

$$
[u-v]_{z}:=d_{T}(u, z)-d_{T}(v, z),
$$

where $d_{T}(u, z)$ is the graph distance between vertices $u$ and $z$. Note that this notion of distance is additive,

$$
[u-v]_{z}+[v-w]_{z}=[u-w]_{z} .
$$

The distance $[u-v]_{z}$ does not change when the reference vertex $z$ is replaced by a vertex $\tilde{z}$, provided that the path from $z$ to $\tilde{z}$ is disjoint from the paths from $z$ to $u$ and from $z$ to $v$. This implies that if $\left(x_{m}\right)_{m \geq 0}$ is a transient path on $T,[u-v]_{x_{m}}$ is constant for $m$ large enough and the following limit is well defined:

$$
[u-v]_{x_{-\infty}}:=\lim _{m \rightarrow \infty}[u-v]_{x_{m}} .
$$

The limit $[u-v]_{x_{-\infty}}$ is called the horodistance of $u$ to $v$, relative to the boundary point $x_{-\infty}$. As observed in [23], and applied to our setting by [19] (see Display (5.1) therein), 
the ergodicity of the environment seen from the particle under $\hat{\mathbb{P}}_{\varepsilon}$ implies that the speed is given as

$$
\lim _{n \rightarrow \infty} \frac{\left|X_{n}\right|}{n}=\hat{\mathbb{E}}_{\varepsilon}\left[\left[X_{1}-X_{0}\right]_{X_{-\infty}}\right]
$$

where the limit holds $\mathbb{P}_{\varepsilon}$-almost surely, or equivalently $\hat{\mathbb{P}}_{\varepsilon}$-almost surely, for any $\varepsilon>0$. Note that the distance $\left[X_{1}-X_{0}\right]_{X_{-\infty}}$ is only well-defined for $\varepsilon>0$. If $\varepsilon=0$, the random walk might start on a tree which is finite when edges with conductance zero are removed. In this case, the random walk in negative time cannot be transient and does not define a boundary point of the tree. We show in the following section that the limit of the speed for $\varepsilon \rightarrow 0$ is given by the corresponding limit of (2.9), on the event that the tree $T_{0}$ formed by edges with positive conductance is infinite, that is,

$$
\lim _{\varepsilon \searrow 0} v\left(\nu, \mu_{\varepsilon}\right)=\hat{\mathbb{E}}_{0}\left[\left[X_{1}-X_{0}\right]_{X_{-\infty}} \mathbb{1}_{\left\{\left|T_{0}\right|=\infty\right\}}\right] .
$$

On the event $\left\{\left|T_{0}\right|=\infty\right\}$ the limit $X_{-\infty}$ is well defined. This implies Theorem 1.2, since

$$
\hat{\mathbb{P}}_{0}\left(\left|T_{0}\right|=\infty\right)=\hat{\mathrm{P}}_{0}\left(\left|T_{0}\right|=\infty\right)
$$

and

$$
\hat{\mathbb{E}}_{0}\left[\left[X_{1}-X_{0}\right]_{X_{-\infty}}|| T_{0} \mid=\infty\right]=v\left(\nu, \mu_{0}\right),
$$

see Remark 4.1 in [19].

\section{Proofs of the main results}

\subsection{Proof of Proposition 1.1}

It was proven by [19, Theorem 4.1], that the speed can be expressed as an expectation of ratios of effective conductances. Let $\mathcal{C}(T)$ be the effective conductance from the root to infinity, then

$$
v(\nu, \mu)=1-\frac{2}{\gamma} \mathrm{E}^{\text {aug }}\left[\xi_{0} \frac{\mathcal{C}\left(T^{*}\right)}{\mathcal{C}(T)}\right],
$$

where $\xi_{0}=\xi\left(\rho, v_{0}\right)$ denotes the conductance of the additional edge in the augmented Galton-Watson tree and $T^{*}$ denotes the subtree composed of the tree rooted at $v_{0}$ and the additional edge $\left(\rho, v_{0}\right)$.

Now, let $\left(\mu_{n}\right)_{n \in \mathbb{N}}$ and $\mu$ be uniformly elliptic, i.e. satisfying (1.2) for a common $\delta>0$, such that $\mu_{n} \rightarrow \mu$ weakly. We denote the law of the augmented Galton-Watson tree by $\mathrm{P}_{n}^{\text {aug }}$ (respectively $\mathrm{P}^{\text {aug }}$ ), if the marginal law of the conductances is given by $\mu_{n}$ (respectively $\mu$ ). Then, (3.1) implies

$$
\begin{aligned}
\lim _{n \rightarrow \infty} v\left(\nu, \mu_{n}\right) & =1-\frac{2}{\gamma} \lim _{n \rightarrow \infty} \mathrm{E}_{n}^{\text {aug }}\left[\xi_{0} \frac{\mathcal{C}\left(T^{*}\right)}{\mathcal{C}(T)}\right] \\
& =1-\frac{2}{\gamma} \lim _{n \rightarrow \infty} \mathrm{E}_{n}^{\text {aug }}\left[\mathrm{E}_{n}^{\text {aug }}\left[\xi_{0} \frac{\mathcal{C}\left(T^{*}\right)}{\mathcal{C}(T)} \mid T\right]\right] \\
& =1-\frac{2}{\gamma} \lim _{n \rightarrow \infty} \mathrm{E}^{\text {aug }}\left[\mathrm{E}_{n}^{\text {aug }}\left[\xi_{0} \frac{\mathcal{C}\left(T^{*}\right)}{\mathcal{C}(T)} \mid T\right]\right],
\end{aligned}
$$

where for the last equality, we used that the distribution of $T$ is independent of $n$. We first show that the sequence of the conditional expectations in (3.2) convergences in distribution,

$$
\mathrm{E}_{n}^{\text {aug }}\left[\xi_{0} \frac{\mathcal{C}\left(T^{*}\right)}{\mathcal{C}(T)} \mid T\right] \underset{n \rightarrow \infty}{\stackrel{d}{\longrightarrow}} \mathrm{E}^{\text {aug }}\left[\xi_{0} \frac{\mathcal{C}\left(T^{*}\right)}{\mathcal{C}(T)} \mid T\right]
$$


The speed of random walk on Galton-Watson trees with vanishing conductances

To see this, we change the probability space by defining a tree $T$ as a subset of the Ulam-Harris tree and labeling each edge of the Ulam-Harris tree (not only the edges in a particular tree) with conductances. This entails that the conductances are independent of the tree, but the considered expectations remain the same. Since $\mu_{n} \rightarrow \mu$ weakly by assumption, the weak convergence $\mathrm{P}_{n} \rightarrow \mathrm{P}$ follows and then also $\mathrm{P}_{n}^{\text {aug }} \rightarrow \mathrm{P}^{\text {aug }}$ by dominated convergence. Hence, (3.3) follows from [12, Corollary 4.1], if the mapping $\xi \mapsto \xi_{0} \frac{\mathcal{C}\left(T^{*}\right)}{\mathcal{C}(T)}$ is bounded and continuous. Boundedness follows from $\mathcal{C}\left(T^{*}\right) \leq \mathcal{C}(T)$ and the uniform ellipticity of $\mu_{n}$. Dirichlet's Principle implies that the effective conductance can be expressed as the minimum of a linear function in $\xi$, thus it is concave. Furthermore, for almost all $T, T^{*}$, the simple random walks on $T, T^{*}$ are transient, which implies that $\xi \mapsto C(T)$ and $\xi \mapsto C\left(T^{*}\right)$ are locally bounded on the convex set $\left\{\left(\xi_{e}\right)_{e}: \xi_{e}>0\right\}$. Therefore (see [27, Section 41]), both effective conductances are continuous on $\left\{\left(\xi_{e}\right)_{e}: \xi_{e}>0\right\}$ for almost all trees, which implies (3.3).

Now, note that the conditional expectation in (3.2) is bounded by $\delta^{-1}$ for all $n$, thus it is uniformly integrable. So, the sequence of conditional expectations converges in expectation and we obtain from (3.2)

$$
\lim _{n \rightarrow \infty} v\left(\nu, \mu_{n}\right)=1-\frac{2}{\gamma} \mathrm{E}^{\mathrm{aug}}\left[\mathrm{E}^{\mathrm{aug}}\left[\xi_{0} \frac{\mathcal{C}\left(T^{*}\right)}{\mathcal{C}(T)} \mid T\right]\right]=1-\frac{2}{\gamma} \mathrm{E}^{\mathrm{aug}}\left[\xi_{0} \frac{\mathcal{C}\left(T^{*}\right)}{\mathcal{C}(T)}\right]=v(\nu, \mu),
$$

which concludes the proof of Proposition 1.1.

\subsection{Proof of Theorem 1.2}

As mentioned in (2.9), for uniformly elliptic environment measures, the speed is the expectation under the invariant measure $\hat{\mathbb{P}}_{\varepsilon}$ of $D_{\infty}=\left[X_{1}-X_{0}\right]_{X_{-\infty}}$. The weak convergence of $\hat{\mathbb{P}}_{\varepsilon}$ does not imply the convergence of the speed, because $D_{\infty}$ is not a continuous function of the trajectory of the bi-infinite random walk. Also, note that since $T_{0}$ might be finite, $D_{\infty}$ is not well-defined for $\varepsilon=0$. On the event $\left\{\left|T_{0}\right|=\infty\right\}$ however, $D_{\infty}$ is well-defined even if $\varepsilon=0$ and we therefore distinguish whether $T_{0}$ is a finite tree or not. For $0<\varepsilon<\delta$, the tree $T_{0}$ is defined as the subgraph formed by the edges with conductance larger than $\varepsilon$ containing the root, see Figure 2. The proof of Theorem 1.2 follows from the two following lemmas.

Lemma 3.1. On the event $\left\{\left|T_{0}\right|=\infty\right\}$, the mean of $D_{\infty}$ converges to its mean under $\hat{\mathbb{P}}_{0}$,

$$
\lim _{\varepsilon \rightarrow 0} \hat{\mathbb{E}}_{\varepsilon}\left[D_{\infty} \mathbb{1}_{\left\{\left|T_{0}\right|=\infty\right\}}\right]=\hat{\mathbb{E}}_{0}\left[D_{\infty} \mathbb{1}_{\left\{\left|T_{0}\right|=\infty\right\}}\right] \text {. }
$$

Lemma 3.2. On the event $\left\{\left|T_{0}\right|<\infty\right\}$, the mean of $D_{\infty}$ vanishes,

$$
\lim _{\varepsilon \rightarrow 0} \hat{\mathbb{E}}_{\varepsilon}\left[D_{\infty} \mathbb{1}_{\left\{\left|T_{0}\right|<\infty\right\}}\right]=0 .
$$

Combining the representation for the speed in (2.9) with Lemma 3.1 and Lemma 3.2, we obtain

$$
\lim _{\varepsilon \rightarrow 0} v\left(\nu, \mu_{\varepsilon}\right)=\lim _{\varepsilon \rightarrow 0} \hat{\mathbb{E}}_{\varepsilon}\left[D_{\infty}\right]=\hat{\mathbb{E}}_{0}\left[D_{\infty} \mathbb{1}_{\left\{\left|T_{0}\right|=\infty\right\}}\right]=\hat{\mathbb{E}}_{0}\left[D_{\infty}|| T_{0} \mid=\infty\right] \hat{\mathrm{P}}_{0}\left(\left|T_{0}\right|=\infty\right)
$$

and as described in the introduction (see also (2.12)), $\hat{\mathbb{E}}_{0}\left[D_{\infty}|| T_{0} \mid=\infty\right]=v\left(\nu, \mu_{0}\right)$.

\subsubsection{Proof of Lemma 3.1}

The main ingredients of the proof are the continuity of $\hat{\mathbb{P}}_{\varepsilon}$ as $\varepsilon \rightarrow 0$ and a transience estimate on infinite subtrees $T_{0}$, which is uniform in $\varepsilon$. 


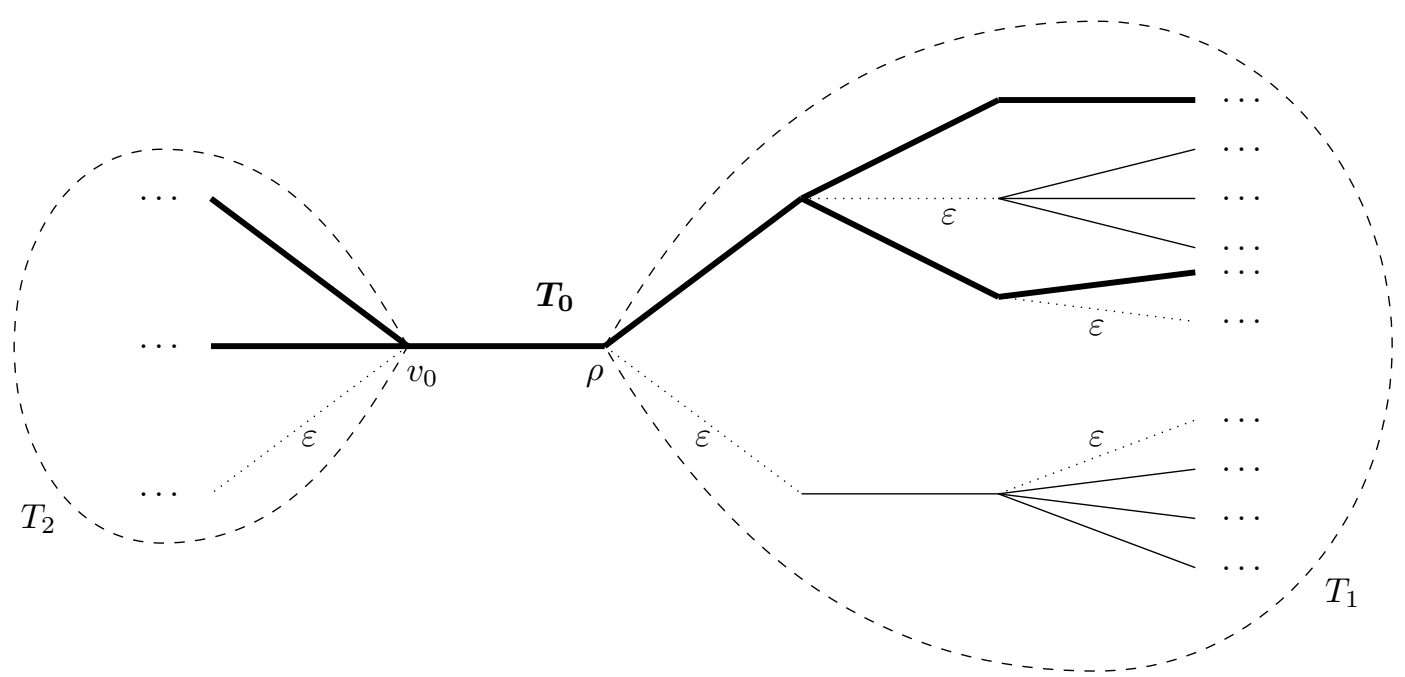

Figure 2: $T_{0}$ is the subgraph formed by the edges with conductance larger than $\varepsilon$ containing the root. $T_{1}$ and $T_{2}$ are independent and identically distributed weighted Galton-Watson trees (see Figure 1) and edges with conductance $\varepsilon$ are indicated by dotted lines.

As an approximation for $D_{\infty}$ which depends only on finitely many coordinates, we introduce

$$
D_{M}:=\left[X_{1}-X_{0}\right]_{X_{-M}}=d_{T}\left(X_{1}, X_{-M}\right)-d_{T}\left(X_{0}, X_{-M}\right) .
$$

Since $D_{M}$ depends only on finitely many coordinates, it is a continuous and uniformly bounded function of the trajectory. Moreover, we approximate $\mathbb{1}_{\left\{\left|T_{0}\right|=\infty\right\}}$ by the indicator functions $\mathbb{1}_{\left\{\left|T_{0}\right|>N\right\}}$, which are continuous functions of the environment. For the limit of the speed we write then

$$
\begin{gathered}
\lim _{\varepsilon \rightarrow 0} \hat{\mathbb{E}}_{\varepsilon}\left[D_{\infty} \mathbb{1}_{\left\{\left|T_{0}\right|=\infty\right\}}\right]=\lim _{\varepsilon \rightarrow 0}\left(\hat{\mathbb{E}}_{\varepsilon}\left[D_{M} \mathbb{1}_{\left\{\left|T_{0}\right|>N\right\}}\right]+\hat{\mathbb{E}}_{\varepsilon}\left[D_{M}\left(\mathbb{1}_{\left\{\left|T_{0}\right|=\infty\right\}}-\mathbb{1}_{\left\{\left|T_{0}\right|>N\right\}}\right)\right]\right. \\
\left.+\hat{\mathbb{E}}_{\varepsilon}\left[\left(D_{\infty}-D_{M}\right) \mathbb{1}_{\left\{\left|T_{0}\right|=\infty\right\}}\right]\right) .
\end{gathered}
$$

By definition (2.8), we have $D_{M} \rightarrow D_{\infty}$ as $M \rightarrow \infty$ almost surely on any infinite tree. The weak convergence in Lemma 2.1 and dominated convergence yield then for the first term on the right hand side of (3.4)

$$
\lim _{M \rightarrow \infty} \lim _{N \rightarrow \infty} \lim _{\varepsilon \rightarrow 0} \hat{\mathbb{E}}_{\varepsilon}\left[D_{M} \mathbb{1}_{\left\{\left|T_{0}\right|>N\right\}}\right]=\lim _{M \rightarrow \infty} \lim _{N \rightarrow \infty} \hat{\mathbb{E}}_{0}\left[D_{M} \mathbb{1}_{\left\{\left|T_{0}\right|>N\right\}}\right]=\hat{\mathbb{E}}_{0}\left[D_{\infty} \mathbb{1}_{\left\{\left|T_{0}\right|=\infty\right\}}\right] .
$$

We can bound the absolute value of the second expectation in (3.4) by

$$
\left|\hat{\mathbb{E}}_{\varepsilon}\left[D_{M}\left(\mathbb{1}_{\left\{\left|T_{0}\right|=\infty\right\}}-\mathbb{1}_{\left\{\left|T_{0}\right|>N\right\}}\right)\right]\right| \leq 2 \hat{\mathrm{E}}_{\varepsilon}\left[\mathbb{1}_{\left\{\left|T_{0}\right|>N\right\}}-\mathbb{1}_{\left\{\left|T_{0}\right|=\infty\right\}}\right] .
$$

Recall the definition of $\hat{\mathrm{P}}_{\varepsilon}$ in (2.2), we have by conditioning on the Galton-Watson tree

$$
\begin{aligned}
\hat{\mathrm{E}}_{\varepsilon}\left[\mathbb{1}_{\left\{\left|T_{0}\right|>N\right\}}-\mathbb{1}_{\left\{\left|T_{0}\right|=\infty\right\}}\right] & =\mathrm{E}_{\varepsilon}^{\text {aug }}\left[\left(\mathbb{1}_{\left\{\left|T_{0}\right|>N\right\}}-\mathbb{1}_{\left\{\left|T_{0}\right|=\infty\right\}}\right) \frac{\pi(\rho)}{\gamma_{\varepsilon} \operatorname{deg}(\rho)}\right] \\
& =\mathrm{E}_{\varepsilon}^{\text {aug }}\left[\left(\mathbb{1}_{\left\{\left|T_{0}\right|>N\right\}}-\mathbb{1}_{\left\{\left|T_{0}\right|=\infty\right\}}\right) \frac{1}{\gamma_{\varepsilon} \operatorname{deg}(\rho)} \mathrm{E}_{\varepsilon}^{\operatorname{aug}}[\pi(\rho) \mid T]\right] \\
& =\mathrm{E}_{\varepsilon}^{\text {aug }}\left[\mathbb{1}_{\left\{\left|T_{0}\right|>N\right\}}-\mathbb{1}_{\left\{\left|T_{0}\right|=\infty\right\}}\right] \\
& =\mathrm{E}_{0}^{\text {aug }}\left[\mathbb{1}_{\left\{\left|T_{0}\right|>N\right\}}-\mathbb{1}_{\left\{\left|T_{0}\right|=\infty\right\}}\right] .
\end{aligned}
$$


The speed of random walk on Galton-Watson trees with vanishing conductances

To see the last equality, observe that the distribution of the indicator functions does not depend on $\varepsilon$, since $T_{0}$ is the subtree formed by the edges with conductance at least $\delta$. From this and (3.6), we obtain

$$
\lim _{N \rightarrow \infty} \lim _{\varepsilon \rightarrow 0}\left|\hat{\mathbb{E}}_{\varepsilon}\left[D_{M}\left(\mathbb{1}_{\left\{\left|T_{0}\right|=\infty\right\}}-\mathbb{1}_{\left\{\left|T_{0}\right|>N\right\}}\right)\right]\right| \leq \lim _{N \rightarrow \infty} 2 \mathrm{E}_{0}^{\text {aug }}\left[\mathbb{1}_{\left\{\left|T_{0}\right|>N\right\}}-\mathbb{1}_{\left\{\left|T_{0}\right|=\infty\right\}}\right]=0 .
$$

In view of (3.4) and the limits (3.5) and (3.7), the proof is completed once we show

$$
\lim _{M \rightarrow \infty} \limsup _{\varepsilon \rightarrow 0} \hat{\mathbb{E}}_{\varepsilon}\left[\left|D_{\infty}-D_{M}\right| \mathbb{1}_{\left\{\left|T_{0}\right|=\infty\right\}}\right]=0 .
$$

In fact, we may replace the event $\left\{\left|T_{0}\right|=\infty\right\}$ by the event $\left\{X_{-M} \in T_{0},\left|T_{0}\right|=\infty\right\}$, since $\left|D_{\infty}-D_{M}\right| \leq 2$ and $\hat{\mathbb{P}}_{\varepsilon}\left(X_{-M} \notin T_{0}\right)$ vanishes as $\varepsilon \rightarrow 0$ for $M$ fixed. On the event that $X_{-k} \neq \rho$ for all $k \geq M$, the $X_{-M}, X_{-M-1}, \ldots$ are all vertices in the same subtree of descendants of $\rho$, which implies $D_{M}=D_{\infty}$. Therefore, we can bound

$\hat{\mathbb{E}}_{\varepsilon}\left[\left|D_{\infty}-D_{M}\right| \mathbb{1}_{\left\{X_{-M} \in T_{0},\left|T_{0}\right|=\infty\right\}}\right] \leq 2 \hat{\mathbb{P}}_{\varepsilon}\left(X_{-k}=\rho\right.$ for some $\left.k \geq M, X_{-M} \in T_{0},\left|T_{0}\right|=\infty\right)$.

We need to show that the right hand side vanishes as $M \rightarrow \infty$, uniformly in $\varepsilon$. That is, we need to bound the return time to the root after a large time $M$, uniformly in $\varepsilon$.

We denote by $\mathcal{C}_{\omega}(v, A)$ the effective conductance between vertex $v$ and a set $A$ of vertices in the environment $\omega$, see [14] or [25]. Let $\eta(A)$ denote the hitting time of the set $A$, then (see [8, Fact 2] or [25, Exercise 2.34]) we have for a random walk starting at $v$

$$
P_{\omega}(\eta(A)<\eta(B)) \leq \frac{\mathcal{C}_{\omega}(v, A)}{\mathcal{C}_{\omega}(v, B)}
$$

This yields in our situation

$$
\hat{P}_{\omega}\left(X_{-k}=\rho \text { for some } k \geq M \mid X_{-M}=v\right) \leq \frac{\mathcal{C}_{\omega}(v, \rho)}{\mathcal{C}_{\omega}(v, \infty)},
$$

which implies

$\hat{\mathbb{P}}_{\varepsilon}\left(X_{-k}=\rho\right.$ for some $\left.k \geq M, X_{-M} \in T_{0},\left|T_{0}\right|=\infty\right) \leq \hat{\mathbb{E}}_{\varepsilon}\left[\frac{\mathcal{C}_{\omega}\left(X_{-M}, \rho\right)}{\mathcal{C}_{\omega}\left(X_{-M}, \infty\right)} \mathbb{1}_{\left\{X_{-M} \in T_{0},\left|T_{0}\right|=\infty\right\}}\right]$

The Cauchy-Schwarz inequality implies the bound

$$
\begin{aligned}
& \hat{\mathbb{P}}_{\varepsilon}\left(X_{-k}=\rho \text { for some } k \geq M, X_{-M} \in T_{0},\left|T_{0}\right|=\infty\right)^{2} \\
& \quad \leq \hat{\mathbb{E}}_{\varepsilon}\left[\mathcal{C}_{\omega}\left(X_{-M}, \rho\right)^{2} \mathbb{1}_{\left\{\left|T_{0}\right|=\infty\right\}}\right] \hat{\mathbb{E}}_{\varepsilon}\left[\mathcal{C}_{\omega}\left(X_{-M}, \infty\right)^{-2} \mathbb{1}_{\left\{X_{-M} \in T_{0},\left|T_{0}\right|=\infty\right\}}\right] .
\end{aligned}
$$

For the first expectation, we note that the effective conductance between the root and $X_{-M}$ depends only on the path between these two vertices, so that for any $N \geq 1$, $\hat{E}_{\omega}\left[\mathcal{C}_{\omega}\left(X_{-M}, \rho\right)\right] \mathbb{1}_{\left\{\left|T_{0}\right|>N\right\}}$ is a continuous function of $\omega$. By the uniform ellipticy assumption (1.2), it is bounded by $(M \delta)^{-1}$. Lemma 2.1 implies then

$$
\begin{aligned}
\limsup _{\varepsilon \rightarrow 0} \hat{\mathbb{E}}_{\varepsilon}\left[\mathcal{C}_{\omega}\left(X_{-M}, \rho\right)^{2} \mathbb{1}_{\left\{\left|T_{0}\right|=\infty\right\}}\right] & \leq \limsup _{\varepsilon \rightarrow 0} \hat{\mathbb{E}}_{\varepsilon}\left[\mathcal{C}_{\omega}\left(X_{-M}, \rho\right)^{2} \mathbb{1}_{\left\{\left|T_{0}\right|>N\right\}}\right] \\
& =\hat{\mathbb{E}}_{0}\left[\mathcal{C}_{\omega}\left(X_{-M}, \rho\right)^{2} \mathbb{1}_{\left\{\left|T_{0}\right|>N\right\}}\right]
\end{aligned}
$$

Letting $N \rightarrow \infty$, we have by monotone convergence

$$
\limsup _{\varepsilon \rightarrow 0} \hat{\mathbb{E}}_{\varepsilon}\left[\mathcal{C}_{\omega}\left(X_{-M}, \rho\right)^{2} \mathbb{1}_{\left\{\left|T_{0}\right|=\infty\right\}}\right] \leq \hat{\mathbb{E}}_{0}\left[\mathcal{C}_{\omega}\left(X_{-M}, \rho\right)^{2} \mathbb{1}_{\left\{\left|T_{0}\right|=\infty\right\}}\right]
$$


Since under $\hat{\mathbb{P}}_{0}$, conditioned on $\left|T_{0}\right|=\infty$, the random walk is transient and the conductance between $X_{-M}$ and $\rho$ is bounded by $\left(\left|X_{-M}\right| \delta\right)^{-1}$, the conductance $\mathcal{C}_{\omega}\left(X_{-M}, \rho\right)$ converges almost surely to zero. Dominated convergence implies then

$$
\lim _{M \rightarrow \infty} \limsup _{\varepsilon \rightarrow 0} \hat{\mathbb{E}}_{\varepsilon}\left[\mathcal{C}_{\omega}\left(X_{-M}, \rho\right)^{2} \mathbb{1}_{\left\{\left|T_{0}\right|=\infty\right\}}\right]=0 .
$$

We now show that the second expectation in (3.12) remains bounded. Let us denote by $\mathcal{R}(v, \infty)=\mathcal{C}_{\omega}(v, \infty)^{-1}$ the effective resistance. Since the process $\left(T, X_{n}, \xi\right)_{n \in \mathbb{Z}}$ is stationary under $\hat{\mathbb{P}}_{\varepsilon}$,

$$
\begin{aligned}
\hat{\mathbb{E}}_{\varepsilon}\left[\mathcal{R}_{\omega}\left(X_{-M}, \infty\right)^{2} \mathbb{1}_{\left\{X_{-M} \in T_{0},\left|T_{0}\right|=\infty\right\}}\right] & =\hat{\mathbb{E}}_{\varepsilon}\left[\mathcal{R}_{\omega}(\rho, \infty)^{2} \mathbb{1}_{\left\{X_{M} \in T_{0},\left|T_{0}\right|=\infty\right\}}\right] \\
& \leq \hat{\mathrm{E}}_{\varepsilon}\left[\mathcal{R}_{\omega}(\rho, \infty)^{2} \mathbb{1}_{\left\{\left|T_{0}\right|=\infty\right\}}\right]
\end{aligned}
$$

By Rayleigh's monotonicity principle, the effective resistance $\mathcal{R}_{\omega}(\rho, \infty)$ increases when all edges with conductance $\varepsilon$ are removed, which implies

$$
\hat{\mathrm{E}}_{\varepsilon}\left[\mathcal{R}_{\omega}(\rho, \infty)^{2} \mathbb{1}_{\left\{\left|T_{0}\right|=\infty\right\}}\right] \leq \hat{\mathrm{E}}_{0}\left[\mathcal{R}_{\omega}(\rho, \infty)^{2} \mathbb{1}_{\left\{\left|T_{0}\right|=\infty\right\}}\right] \leq \hat{\mathrm{E}}_{0}\left[\mathcal{R}_{\omega}(\rho, \infty)^{2}|| T_{0} \mid=\infty\right]
$$

By the Harris decomposition (see [22, Proposition 4.10]), the tree $T_{0}$, conditioned on $\left|T_{0}\right|=\infty$, may be decomposed into a backbone tree without leaves and with finite trees attached to it. The effective resistance $\mathcal{R}_{\omega}(\rho, \infty)$ only depends on the backbone tree, which is again a supercritical Galton-Watson tree. We may then apply Lemma 3.3 below, which shows that the second moment in (3.17) is finite.

Together with (3.15), this implies that the upper bound in (3.12) vanishes when first $\varepsilon$ tends to zero and then $M$ to infinity. This yields (3.8) and concludes the proof of the lemma.

The following lemma shows the moment bound needed for the proof of Lemma 3.2. It shows that the effective resistance of Galton-Watson trees has finite moments of any order, which might be of independent interest.

Lemma 3.3. Let $T$ be a supercritical Galton-Watson tree without leaves and uniformly elliptic conductances. Then for all $p>0$

$$
\mathrm{E}\left[\mathcal{R}_{\omega}(\rho, \infty)^{p}\right]<\infty .
$$

Proof. Let $G_{n}(T)=\{v \in T:|v|=n\}$ be the $n$-th generation of the tree $T$. We have

$$
\mathcal{R}_{\omega}(\rho, \infty)=\lim _{n \rightarrow \infty} \mathcal{R}_{\omega}\left(\rho, G_{n}(T)\right) .
$$

According to the assumption of the uniformly ellipticity of the conductances there exists some $\delta>0$ such that $\xi(e) \geq \delta$ almost surely. By Rayleigh's monotonicity principle, the effective resistance $\mathcal{R}_{\omega}\left(\rho, G_{n}(T)\right)$ increases when the conductances of all edges are reduced to $\delta$. Let $N$ denote the number of offspring of the root and $v_{1}, \ldots, v_{N}$ be the vertices of the first generation. Moreover, call $T_{v}$ the subtree of $T$ rooted at $v$ composed of $v$ and all its descendants. Using the Parallel Law and the Series Law, we obtain the following recursion

$$
\mathcal{R}_{\omega}\left(\rho, G_{n}(T)\right) \leq \mathcal{R}_{\left(T, \rho,(\delta)_{e}\right)}\left(\rho, G_{n}(T)\right)=\left(\sum_{i=1}^{N} \frac{1}{\delta^{-1}+\mathcal{R}_{\left(T_{v_{i}}, \rho,(\delta)_{e}\right)}\left(v_{i}, G_{n-1}\left(T_{v_{i}}\right)\right)}\right)^{-1} .
$$

For the effective resistance between $v$ and $G_{n}\left(T_{v}\right)$ we write $\mathcal{R}_{n}=\mathcal{R}_{\left(T_{v}, v,(\delta)_{e}\right)}\left(v, G_{n}\left(T_{v}\right)\right)$. Since the subtrees $T_{v_{1}}, \ldots T_{v_{N}}$ are independent Galton-Watson trees, we obtain the 
The speed of random walk on Galton-Watson trees with vanishing conductances

following stochastic domination

$$
\mathcal{R}_{n} \preccurlyeq\left(\frac{1}{\delta^{-1}+\mathcal{R}_{n-1}^{(1)}}+\cdots+\frac{1}{\delta^{-1}+\mathcal{R}_{n-1}^{(N)}}\right)^{-1}
$$

where $\mathcal{R}_{n-1}^{(1)}, \ldots, \mathcal{R}_{n-1}^{(N)}$ denote independent copies of $\mathcal{R}_{n-1}$. Bounding the harmonic mean by the arithmetic mean gives rise to

$$
\mathcal{R}_{n} \preccurlyeq \frac{1}{N^{2}} \sum_{i=1}^{N}\left(\delta^{-1}+\mathcal{R}_{n-1}^{(i)}\right) .
$$

Now, write $\mathcal{R}_{\infty}=\mathcal{R}_{\left(T_{v}, v,(\delta)_{e}\right)}(v, \infty)=\lim _{n \rightarrow \infty} \mathcal{R}_{n}$ for the effective resistance between $v$ and infinity. From [23, Lemma 9.1] follows, that the mean resistance $\mathrm{E}\left[\mathcal{R}_{\infty}\right]$ is bounded. In the following, we show by induction that all the higher moments exist as well. Therefore, suppose that for a given $m \in \mathbb{N}$ the moment $\mathrm{E}\left[\mathcal{R}_{\infty}^{m-1}\right]$ is finite. Using the stochastic domination in (3.18), we have

$$
\begin{aligned}
\mathrm{E}\left[\mathcal{R}_{n}^{m}\right] & \leq \mathrm{E}\left[\left((\delta N)^{-1}+N^{-2} \sum_{i=1}^{N} \mathcal{R}_{n-1}^{(i)}\right)^{m}\right] \\
& =\mathrm{E}\left[\sum_{k=0}^{m}\left(\begin{array}{c}
m \\
k
\end{array}\right)(\delta N)^{k-m} N^{-k}\left(N^{-1} \sum_{i=1}^{N} \mathcal{R}_{n-1}^{(i)}\right)^{k}\right] .
\end{aligned}
$$

Applying Jensen's inequality gives the following upper bound,

$$
\begin{aligned}
\mathrm{E}\left[\mathcal{R}_{n}^{m}\right] & \leq \sum_{k=0}^{m}\left(\begin{array}{c}
m \\
k
\end{array}\right) \delta^{k-m} \mathrm{E}\left[N^{-m-1} \sum_{i=1}^{N}\left(\mathcal{R}_{n-1}^{(i)}\right)^{k}\right] \\
& =\sum_{k=0}^{m}\left(\begin{array}{c}
m \\
k
\end{array}\right) \delta^{k-m} \mathrm{E}\left[N^{-m-1} \sum_{i=1}^{N} \mathrm{E}\left[\left(\mathcal{R}_{n-1}^{(i)}\right)^{k} \mid N\right]\right] \\
& =\sum_{k=0}^{m-1}\left(\begin{array}{c}
m \\
k
\end{array}\right) \delta^{k-m} \mathrm{E}\left[N^{-m}\right] \mathrm{E}\left[\mathcal{R}_{n-1}^{k}\right]+\mathrm{E}\left[N^{-m}\right] \mathrm{E}\left[\mathcal{R}_{n-1}^{m}\right] \\
& \leq \sum_{k=0}^{m-1}\left(\begin{array}{c}
m \\
k
\end{array}\right) \delta^{k-m} \mathrm{E}\left[N^{-m}\right] \mathrm{E}\left[\mathcal{R}_{\infty}^{k}\right]+\mathrm{E}\left[N^{-m}\right] \mathrm{E}\left[\mathcal{R}_{n-1}^{m}\right]
\end{aligned}
$$

where we used the monotonicity of the effective resistance in the last inequality. More precisely, we have $\mathcal{R}_{n} \leq \mathcal{R}_{\infty}$ for all $n$. The finiteness of $\mathrm{E}\left[\mathcal{R}_{\infty}^{k}\right]$ for $k=0, \ldots, m-1$ follows from the induction hypothesis.

A vanishing resistance between two vertices corresponds to shorting them together, so that they behave as if they were a single vertex. Consistently to this interpretation, we define $\mathcal{R}_{0}=0$. Hence, we have a recursion of the from $x_{n} \leq a+b x_{n-1}, x_{0}=0$, for some $a, b>0$. Iterating this gives rise to $x_{n} \leq a \sum_{k=0}^{n-1} b^{k}$. In our setting, iterating (3.19) leads to

$$
\mathrm{E}\left[\mathcal{R}_{n}^{m}\right] \leq\left(\sum_{k=0}^{m-1}\left(\begin{array}{c}
m \\
k
\end{array}\right) \delta^{k-m} \mathrm{E}\left[N^{-m}\right] \mathrm{E}\left[\mathcal{R}_{\infty}^{k}\right]\right) \sum_{k=0}^{n-1} \mathrm{E}\left[N^{-m}\right]^{k}
$$

Since the absolute value of $\mathrm{E}\left[N^{-m}\right]$ is less than one, (3.20) converges and

$$
\begin{aligned}
\mathrm{E}\left[\mathcal{R}_{\omega}(\rho, \infty)^{m}\right] & \leq \mathrm{E}\left[\mathcal{R}_{\infty}^{m}\right]=\lim _{n \rightarrow \infty} \mathrm{E}\left[\mathcal{R}_{n}^{m}\right] \\
& \leq\left(\sum_{k=0}^{m-1}\left(\begin{array}{c}
m \\
k
\end{array}\right) \delta^{k-m} \mathrm{E}\left[N^{-m}\right] \mathrm{E}\left[\mathcal{R}_{\infty}^{k}\right]\right) \cdot \frac{1}{1-\mathrm{E}\left[N^{-m}\right]}<\infty
\end{aligned}
$$

which completes the proof. 
The speed of random walk on Galton-Watson trees with vanishing conductances

\subsubsection{Proof of Lemma 3.2}

The main ingredients of this proof are the stationarity and the ergodicity of the process $\left(T, X_{n}, \xi\right)_{n \in \mathbb{Z}}$ under $\hat{\mathbb{P}}_{\varepsilon}$. By the ergodic theorem we have

$$
\hat{\mathbb{E}}_{\varepsilon}\left[\left[X_{1}-X_{0}\right]_{X_{-\infty}} \mathbb{1}_{\left\{\left|T_{0}\right|<\infty\right\}}\right]=\lim _{n \rightarrow \infty} \frac{1}{n} \sum_{k=0}^{n-1}\left[X_{k+1}-X_{k}\right]_{X_{-\infty}} \mathbb{1}_{\left\{\left|T_{0}\left(X_{k}\right)\right|<\infty\right\}}
$$

almost surely, where $T_{0}(v)$ is the subtree formed by edges with conductance larger than $\varepsilon$ and containing $v$. As the sum can only increase when the random walk moves on finite trees, let us define the points in time, when the random walk enters and leaves finite trees,

$$
\begin{aligned}
b_{1} & =\inf \left\{n \geq 1: X_{n} \notin T_{0}\left(X_{0}\right)\right\}, \\
a_{k+1} & =\inf \left\{n \geq b_{k}:\left|T_{0}\left(X_{n}\right)\right|<\infty\right\}, \quad k \in \mathbb{N}, \\
b_{k+1} & =\inf \left\{n \geq a_{k}: X_{n} \notin T_{0}\left(X_{a_{k}}\right)\right\}, \quad k \in \mathbb{N} .
\end{aligned}
$$

Every time the random walk reaches a new record distance from the root, it has a positive probability to encounter and travere an $\varepsilon$-edge to a vertex $x$ such that $T_{0}(x)$ is finite. Every finite $T_{0}(x)$ is left after a finite amount of time, which implies that the sequence $\left(b_{n}\right)_{n}$ is unbounded. Since the limit in (3.21) exists, it is equal to the limit along the subsequence, which consists of the times when the random walk is located in a finite tree,

$$
\begin{aligned}
\hat{\mathbb{E}}_{\varepsilon}\left[\left[X_{1}-X_{0}\right]_{X_{-\infty}} \mathbb{1}_{\left\{\left|T_{0}\right|<\infty\right\}}\right]= & \lim _{n \rightarrow \infty} \frac{1}{b_{n}} \sum_{k=0}^{b_{1}-1}\left[X_{k+1}-X_{k}\right]_{X_{-\infty}} \mathbb{1}_{\left\{\left|T_{0}\left(X_{k}\right)\right|<\infty\right\}} \\
& +\lim _{n \rightarrow \infty} \frac{1}{b_{n}} \sum_{k=2}^{n} \sum_{i=a_{k}}^{b_{k}-1}\left[X_{i+1}-X_{i}\right]_{X_{-\infty}} .
\end{aligned}
$$

We note that the first limit in (3.22) vanishes almost surely. Now, let $L_{k}$ denote the time that the random walk spends in the tree $T_{0}\left(X_{k}\right)$ until leaving it, that is

$$
\begin{aligned}
L_{k}= & \sup \left\{n \in \mathbb{N}: X_{k+1}, \ldots, X_{k+n} \in T_{0}\left(X_{k}\right)\right\} \\
& +\sup \left\{n \in \mathbb{N}: X_{k-1}, \ldots, X_{k-n} \in T_{0}\left(X_{k}\right)\right\}+1 .
\end{aligned}
$$

Bounding the distance from the root that the random walks can gain on the $k$-th finite tree by its number of vertices, we obtain the following upper bound for the second limit in (3.22)

$$
\begin{aligned}
\lim _{n \rightarrow \infty} \frac{1}{b_{n}} \sum_{k=2}^{n} \sum_{i=a_{k}}^{b_{k}-1}\left[X_{i+1}-X_{i}\right]_{X_{-\infty}} & \leq \lim _{n \rightarrow \infty} \frac{1}{b_{n}} \sum_{k=2}^{n}\left|T_{0}\left(X_{a_{k}}\right)\right| \\
& =\lim _{n \rightarrow \infty} \frac{1}{b_{n}} \sum_{k=2}^{n} \sum_{i=a_{k}}^{b_{k}-1} \frac{1}{L_{i}}\left|T_{0}\left(X_{i}\right)\right| \\
& =\lim _{n \rightarrow \infty} \frac{1}{b_{n}} \sum_{k=a_{2}}^{b_{n}-1} \frac{1}{L_{k}}\left|T_{0}\left(X_{k}\right)\right| \mathbb{1}_{\left\{\left|T_{0}\left(X_{k}\right)\right|<\infty\right\}} \\
& \leq \lim _{n \rightarrow \infty} \frac{1}{b_{n}} \sum_{k=0}^{b_{n}-1} \frac{1}{L_{k}}\left|T_{0}\left(X_{k}\right)\right| \mathbb{1}_{\left\{\left|T_{0}\left(X_{k}\right)\right|<\infty\right\}}
\end{aligned}
$$


By the ergodic theorem the averages in (3.23) converge almost surely to their mean

$$
\lim _{n \rightarrow \infty} \frac{1}{b_{n}} \sum_{k=0}^{b_{n}-1} \frac{1}{L_{k}}\left|T_{0}\left(X_{k}\right)\right| \mathbb{1}_{\left\{\left|T_{0}\left(X_{k}\right)\right|<\infty\right\}}=\hat{\mathbb{E}}_{\varepsilon}\left[\frac{1}{L_{0}}\left|T_{0}\left(X_{0}\right)\right| \mathbb{1}_{\left\{\left|T_{0}\left(X_{0}\right)\right|<\infty\right\}}\right] .
$$

Applying the Cauchy-Schwarz inequality gives rise to the following bound

$$
\hat{\mathbb{E}}_{\varepsilon}\left[\frac{1}{L_{0}}\left|T_{0}\left(X_{0}\right)\right| \mathbb{1}_{\left\{\left|T_{0}\left(X_{0}\right)\right|<\infty\right\}}\right]^{2} \leq \hat{\mathbb{E}}_{\varepsilon}\left[\frac{1}{L_{0}^{2}}\right] \hat{\mathbb{E}}_{\varepsilon}\left[\left|T_{0}\left(X_{0}\right)\right|^{2} \mathbb{1}_{\left\{\left|T_{0}\left(X_{0}\right)\right|<\infty\right\}}\right] .
$$

Since $L_{0} \geq b_{1}$, we can bound the first expectation in (3.24) by

$$
\hat{\mathbb{E}}_{\varepsilon}\left[\frac{1}{L_{0}^{2}}\right] \leq \hat{\mathbb{E}}_{\varepsilon}\left[\frac{1}{b_{1}^{2}}\right]=\sum_{k=0}^{\infty} \frac{1}{k^{2}} \hat{\mathbb{P}}_{\varepsilon}\left(b_{1}=k\right) .
$$

Dominated convergence implies

$$
\lim _{\varepsilon \rightarrow 0} \hat{\mathbb{E}}_{\varepsilon}\left[\frac{1}{L_{0}^{2}}\right] \leq \sum_{k=0}^{\infty} \frac{1}{k^{2}} \lim _{\varepsilon \rightarrow 0} \hat{\mathbb{P}}_{\varepsilon}\left(b_{1}=k\right) .
$$

By definition of $b_{1}$, we have

$$
\begin{aligned}
\hat{\mathbb{P}}_{\varepsilon}\left(b_{1}=k\right) & =\hat{\mathbb{P}}_{\varepsilon}\left(X_{0}, \ldots, X_{k-1} \in T_{0}, X_{k} \notin T_{0}\right) \\
& \leq \hat{\mathbb{P}}_{\varepsilon}\left(X_{0}, \ldots, X_{k-1} \in T_{0}, X_{k} \notin T_{0},\left|T_{0}\right|>1\right)+\hat{\mathbb{P}}_{\varepsilon}\left(\left|T_{0}\right|=1\right) .
\end{aligned}
$$

We distinguish whether the tree $T_{0}$ consists only of the root, because in that case the random walk can only leave $T_{0}$ in the first step. But this case occurs with vanishing probability as $\varepsilon \rightarrow 0$, due to

$$
\hat{\mathbb{P}}_{\varepsilon}\left(\left|T_{0}\right|=1\right)=\frac{1}{\gamma} \int \frac{\pi(\rho)}{\operatorname{deg}(\rho)} \mathbb{1}_{\left\{\left|T_{0}\right|=1\right\}} \mathrm{dP}_{\varepsilon}^{\text {aug }}=\frac{1}{\gamma} \int \frac{\varepsilon \operatorname{deg}(\rho)}{\operatorname{deg}(\rho)} \mathrm{dP}_{\varepsilon}^{\text {aug }}=\frac{\varepsilon}{\gamma} .
$$

Now, we bound the first probability in (3.26) as follows

$$
\begin{aligned}
& \hat{\mathbb{P}}_{\varepsilon}\left(X_{0}, \ldots, X_{k-1} \in T_{0}, X_{k} \notin T_{0},\left|T_{0}\right|>1\right) \\
& \quad=\sum_{i=1}^{\infty} \hat{\mathbb{P}}_{\varepsilon}\left(X_{0}, \ldots, X_{k-1} \in T_{0}, X_{k} \notin T_{0},\left|T_{0}\right|>1 \mid \operatorname{deg}\left(X_{k-1}\right)=i\right) \hat{\mathbb{P}}_{\varepsilon}\left(\operatorname{deg}\left(X_{k-1}\right)=i\right) \\
& \quad \leq \sum_{i=1}^{\infty} p_{i-1} \hat{\mathbb{P}}_{\varepsilon}\left(X_{k} \notin T_{0}\left|X_{0}, \ldots, X_{k-1} \in T_{0},\right| T_{0} \mid>1, \operatorname{deg}\left(X_{k-1}\right)=i\right) \\
& \quad \leq \sum_{i=1}^{\infty} p_{i-1} \frac{(i-1) \varepsilon}{(i-1) \varepsilon+\delta}
\end{aligned}
$$

To see the last inequality, observe that there is at least one edge with conductance greater than $\delta$ adjacent to the vertex, where the random walk is located after $k-1$ steps. Decreasing the conductance of the other adjoining edges to $\varepsilon$ implies the bound we used above. Plugging (3.27) and (3.28) in (3.26), dominated convergence implies

$$
\lim _{\varepsilon \rightarrow 0} \hat{\mathbb{P}}_{\varepsilon}\left(b_{1}=k\right) \leq \sum_{i=1}^{\infty} p_{i-1} \lim _{\varepsilon \rightarrow 0} \frac{(i-1) \varepsilon}{(i-1) \varepsilon+\delta}+\lim _{\varepsilon \rightarrow 0} \frac{\varepsilon}{\gamma}=0 .
$$

Hence, due to (3.25), the second inverse moment of $L_{0}$ vanishes as $\varepsilon \rightarrow 0$.

To complete the proof, we show that the second expectation in (3.24) remains bounded. By the duality principle for branching processes (see [2, Section 12]), the tree 
The speed of random walk on Galton-Watson trees with vanishing conductances

$T_{0}$, conditioned on extinction, is a Galton-Watson tree. Since the second moment of the offspring law exists, Lemma 3.4 below shows that the second moment in (3.24) is finite, which concludes the proof of Lemma 3.2.

The following lemma is needed for the proof of Lemma 3.2. It gives a formula to compute the second moment of the total progeny of a branching process. The formula is also given in [26], we include a proof for completeness.

Lemma 3.4. For a branching process with independent and identically distributed offspring $X$ having offspring mean $E[X]=m_{1}<1$ and finite second moment $E\left[X^{2}\right]=$ $m_{2}<\infty$, the second moment of the total progeny is finite, precisely

$$
\mathrm{E}\left[T^{2}\right]=\frac{m_{2}-m_{1}^{2}-m_{1}+1}{\left(1-m_{1}\right)^{3}} .
$$

Proof. Let $Z_{n}$ denote the size of the $n$-th generation, that is

$$
Z_{0}=1, \quad Z_{n}=\sum_{i=1}^{Z_{n-1}} X_{n, i}
$$

where $\left(X_{n, i}\right)_{i, n \in \mathbb{N}}$ is a doubly infinite array of independent and identically distributed random variables with $X_{n, i} \sim X$ for all $n, i \in \mathbb{N}$. Then, the total progeny of the branching process is given by $T=Z_{0}+Z_{1}+\ldots$. Monotone convergence implies the following respresentation of second moment of the total progeny

$$
\mathrm{E}\left[T^{2}\right]=\sum_{n=0}^{\infty} \mathrm{E}\left[Z_{n}^{2}\right]+2 \sum_{m=0}^{\infty} \sum_{n=m+1}^{\infty} \mathrm{E}\left[Z_{m} Z_{n}\right]
$$

We need to compute the second moment of the generation sizes and the mixed moments. A straightforward calculation shows that

$$
\mathrm{E}\left[Z_{n}^{2}\right]=m_{2} m_{1}^{n-1}-m_{1}^{n+1}+m_{1}^{2} \mathrm{E}\left[Z_{n-1}^{2}\right]
$$

Iterating (3.30) leads to (see also [2, Section 2])

$$
\mathrm{E}\left[Z_{n}^{2}\right]=\frac{m_{2} m_{1}^{n-1}\left(1-m_{1}^{n}\right)-m_{1}^{n+1}+m_{1}^{2 n}}{1-m_{1}} .
$$

Next, we derive a recurrence equation for the mixed moments. Using the independence of $\left(X_{n, i}\right)_{i \in \mathbb{N}}$ and $Z_{n-1}$, we obtain for $n>m$

$$
\begin{aligned}
\mathrm{E}\left[Z_{m} Z_{n}\right] & =\mathrm{E}\left[Z_{m} \sum_{i=1}^{Z_{n-1}} X_{n, i}\right]=\mathrm{E}\left[Z_{m} \sum_{i=1}^{Z_{n-1}} \mathrm{E}\left[X_{n, i} \mid Z_{1}, \ldots, Z_{n-1}\right]\right] \\
& =\mathrm{E}\left[Z_{m} \sum_{i=1}^{Z_{n-1}} \mathrm{E}\left[X_{n, i}\right]\right]=\mathrm{E}\left[Z_{m} Z_{n-1}\right] \mathrm{E}[X]=\mu \mathrm{E}\left[Z_{m} Z_{n-1}\right] .
\end{aligned}
$$

Iterating this gives rise to

$$
\mathrm{E}\left[Z_{m} Z_{n}\right]=m_{1}^{n-m} \mathrm{E}\left[Z_{m}^{2}\right]
$$


for $n>m$. Plugging this in (3.29), we obtain

$$
\begin{aligned}
E\left[T^{2}\right] & =\sum_{n=0}^{\infty} \mathrm{E}\left[Z_{n}^{2}\right]+2 \sum_{m=0}^{\infty} m_{1}^{-m} \mathrm{E}\left[Z_{m}^{2}\right] \sum_{n=m+1}^{\infty} m_{1}^{n} \\
& =\sum_{n=0}^{\infty} \mathrm{E}\left[Z_{n}^{2}\right]+\frac{2 m_{1}}{1-m_{1}} \sum_{n=0}^{\infty} \mathrm{E}\left[Z_{n}^{2}\right] \\
& =\frac{1+m_{1}}{1-m_{1}} \sum_{n=0}^{\infty} \mathrm{E}\left[Z_{n}^{2}\right] .
\end{aligned}
$$

Together with (3.31), we have

$$
E\left[T^{2}\right]=\frac{1+m_{1}}{\left(1-m_{1}\right)^{2}} \sum_{n=0}^{\infty}\left(m_{2} m_{1}^{n-1}\left(1-m_{1}^{n}\right)-m_{1}^{n+1}+m_{1}^{2 n}\right),
$$

which is finite, since $m_{1}<1$ holds by assumption. More precisely, we have

$$
\begin{aligned}
E\left[T^{2}\right] & =\frac{1+m_{1}}{\left(1-m_{1}\right)^{2}}\left(\frac{m_{2}}{m_{1}\left(1-m_{1}\right)}-\frac{m_{2}}{m_{1}\left(1-m_{1}^{2}\right)}-\frac{m_{1}}{1-m_{1}}+\frac{1}{1-m_{1}^{2}}\right) \\
& =\frac{m_{2}-m_{1}^{2}-m_{1}+1}{\left(1-m_{1}\right)^{3}} .
\end{aligned}
$$

\subsection{Proof of Lemma 2.1}

We first show the weak convergence of $\hat{\mathbb{P}}_{\varepsilon}$ on $\mathcal{G} \otimes \mathcal{F}_{m}$, where $\mathcal{G}$ is the $\sigma$-algebra on $\Omega$ and $\mathcal{F}_{m}=\sigma\left(X_{-m}, \ldots, X_{m}\right)$, recall the definitions following (1.4) and (2.2). Note that since $\hat{\mathrm{P}}_{\varepsilon}$ is the marginal law of $\omega$ under $\hat{\mathbb{P}}_{\varepsilon}$, the weak convergence of $\hat{\mathrm{P}}_{\varepsilon}$ is implied by the one of $\hat{\mathbb{P}}_{\varepsilon}$. Let $f$ be a bounded and continuous function, measurable with respect to $\mathcal{G} \otimes \mathcal{F}_{m}$. Let us denote by $T_{\mid B_{m}(\rho)}$ the subgraph of $T$ consisting of the vertices of $T$ with graph distance to $\rho$ at most $m$, and the edges connecting those vertices. We have

$$
\begin{aligned}
\left|\int f \mathrm{~d} \hat{\mathbb{P}}_{\varepsilon}-\int f \mathrm{~d} \hat{\mathbb{P}}_{0}\right| \leq & \left|\int f \mathbb{1}_{\left\{\left|T_{\mid B_{m}(\rho)}\right| \leq M\right\}} \mathrm{d} \hat{\mathbb{P}}_{\varepsilon}-\int f \mathbb{1}_{\left\{\left|T_{\mid B m}(\rho)\right| \leq M\right\}} \mathrm{d} \hat{\mathbb{P}}_{0}\right| \\
& +\left|\int f \mathbb{1}_{\left\{\left|T_{\mid B_{m}(\rho)}\right|>M\right\}} \mathrm{d} \hat{\mathbb{P}}_{\varepsilon}\right|+\left|\int f \mathbb{1}_{\left\{\left|T_{\mid B_{m}(\rho)}\right|>M\right\}} \mathrm{d} \hat{\mathbb{P}}_{0}\right|
\end{aligned}
$$

Using the Doob-Dynkin lemma we have $f\left(\omega,\left(x_{n}\right)_{n \in \mathbb{Z}}\right)=g\left(\omega,\left(x_{-m}, \ldots, x_{m}\right)\right)$ for some measurable function $g$. If $\left|T_{\mid B_{m}(\rho)}\right| \leq M$ holds, the number of possible trajectories on $T_{\mid B_{m}(\rho)}$ of length $m$ is finite, and we can write the first integral of (3.32) as the finite sum

$$
\begin{aligned}
& \int f\left(\omega,\left(x_{n}\right)_{n \in \mathbb{Z}}\right) \mathbb{1}_{\left\{\left|T_{\mid B_{m}(\rho)}\right| \leq M\right\}} \mathrm{d} \hat{\mathbb{P}}_{\varepsilon} \\
& =\sum_{x_{-m}, \ldots, x_{m}} \int g\left(\left(x_{-m}, \ldots, x_{m}\right), \omega\right) \mathbb{1}_{\left\{\left|T_{\mid B_{m}(\rho)}\right| \leq M\right\}} \\
& \quad \times \frac{\pi(\rho)}{\gamma_{\varepsilon} \operatorname{deg}(\rho)} \hat{P}_{\omega}\left(X_{-m}=x_{-m}, \ldots, X_{m}=x_{m}\right) \operatorname{dP}_{\varepsilon}^{\operatorname{aug}}(\omega),
\end{aligned}
$$

recall the definition of $\hat{\mathrm{P}}_{\varepsilon}$ in (2.2).

Under $\mathrm{P}_{\varepsilon}$, and also under $\mathrm{P}_{\varepsilon}^{\text {aug }}$, the conductances are i.i.d. conditioned on the tree $T$, with marginal law $\mu_{\varepsilon}$. Since $\mu_{\varepsilon} \rightarrow \mu_{0}$ weakly, the weak convergence $\mathrm{P}_{\varepsilon} \rightarrow \mathrm{P}_{0}$ follows and then also $\mathrm{P}_{\varepsilon}^{\text {aug }} \rightarrow \mathrm{P}_{0}^{\text {aug }}$ by dominated convergence. This implies the convergence of the integrals in (3.33), provided that the integrands are continuous and bounded functions. To verify this, we first look at the mapping $\omega \mapsto \frac{\pi(\rho)}{\operatorname{deg}(\rho)} \hat{P}_{\omega}\left(X_{-m}=x_{-m}, \ldots, X_{m}=x_{m}\right)$. 
The speed of random walk on Galton-Watson trees with vanishing conductances

Let $\omega^{(n)}=\left(T^{(n)}, \rho^{(n)}, \xi^{(n)}\right)$ be a sequence of environments that converges to $\omega=$ $(T, \rho, \xi)$ for $n \rightarrow \infty$, that is, $\left(T_{\mid B_{m}\left(\rho^{(n)}\right)}^{(n)}, \rho^{(n)}\right)=\left(T_{\mid B_{m}(\rho)}, \rho\right)$ for $n$ big enough and

$$
\left(\xi^{(n)}(e)\right)_{e \in \mathcal{E}\left(T_{\mid B_{m}(\rho)}\right)} \rightarrow(\xi(e))_{e \in \mathcal{E}\left(T_{\mid B_{m}(\rho)}\right)} .
$$

For a valid trajectory $x_{-m}, \ldots, x_{m}$ (in particular, $x_{0}=\rho$ ), we have

$$
\begin{aligned}
& \hat{P}_{\omega^{(n)}}\left(X_{-m}=x_{-m}, \ldots, X_{m}=x_{m}\right) \\
& \quad=P_{\omega^{(n)}}\left(X_{0}=x_{0}, \ldots, X_{m}=x_{m}\right) P_{\omega^{(n)}}\left(X_{0}=x_{0}, \ldots, X_{-m}=x_{-m}\right) \\
& \quad=\prod_{k=1}^{m} \frac{\xi^{(n)}\left(x_{k-1}, x_{k}\right)}{\sum_{v \sim x_{k-1}} \xi^{(n)}\left(x_{k-1}, v\right)} \prod_{k=1}^{m} \frac{\xi^{(n)}\left(x_{-(k-1)}, x_{-k}\right)}{\sum_{v \sim x_{-(k-1)}} \xi^{(n)}\left(x_{-(k-1)}, v\right)} .
\end{aligned}
$$

Suppose that the sums $\sum_{v \sim x_{k-1}} \xi\left(x_{k-1}, v\right)$ and $\sum_{v \sim x_{-(k-1)}} \xi\left(x_{-(k-1)}, v\right)$ are strictly positive for all $k$ appearing in the products. In this case, due to the convergence of $\xi^{(n)}$ we easily obtain

$\frac{\pi\left(\rho^{(n)}\right)}{\operatorname{deg}\left(\rho^{(n)}\right)} \hat{P}_{\omega(n)}\left(X_{-m}=x_{-m}, \ldots, X_{m}=x_{m}\right) \underset{n \rightarrow \infty}{\longrightarrow} \frac{\pi(\rho)}{\operatorname{deg}(\rho)} \hat{P}_{\omega}\left(X_{-m}=x_{-m}, \ldots, X_{m}=x_{m}\right)$.

Now, suppose that there exists a $x_{k-1}$ along the path in positive time with $\sum_{v \sim x_{k-1}} \xi\left(x_{k-1}, v\right)=0$. We define

$$
k_{0}=\min \left\{k: \sum_{v \sim x_{k-1}} \xi\left(x_{k-1}, v\right)=0\right\}
$$

as the first time that the sum is zero. If $k_{0}>1$, we notice that the conductance $\xi\left(x_{k_{0}-2}, x_{k_{0}-1}\right)$ is equal to zero, which implies that $\xi^{(n)}\left(x_{k_{0}-2}, x_{k_{0}-1}\right)$ converges to zero. Using that by definition of $k_{0}$ the sum $\sum_{v \sim x_{k_{0}-2}} \xi\left(x_{k_{0}-2}, v\right)$ is strictly positive, we obtain

$$
0 \leq \prod_{k=1}^{m} \frac{\xi^{(n)}\left(x_{k-1}, x_{k}\right)}{\sum_{v \sim x_{k-1}} \xi^{(n)}\left(x_{k-1}, v\right)} \leq \frac{\xi^{(n)}\left(x_{k_{0}-2}, x_{k_{0}-1}\right)}{\sum_{v \sim x_{k_{0}-2}} \xi^{(n)}\left(x_{k_{0}-2}, v\right)} \underset{n \rightarrow \infty}{\longrightarrow} 0
$$

If $k_{0}=1$, we have $\sum_{v \sim \rho} \xi(\rho, v)=\pi(\rho)=0$. In particular, we have $\xi^{(n)}\left(\rho, x_{1}\right) \rightarrow \xi\left(\rho, x_{1}\right)=$ 0 and therefore

$$
\begin{aligned}
0 & \leq \frac{\pi\left(\rho^{(n)}\right)}{\operatorname{deg}\left(\rho^{(n)}\right)} \prod_{k=1}^{m} \frac{\xi^{(n)}\left(x_{k-1}, x_{k}\right)}{\sum_{v \sim x_{k-1}} \xi^{(n)}\left(x_{k-1}, v\right)} \\
& \leq \frac{\pi\left(\rho^{(n)}\right)}{\operatorname{deg}\left(\rho^{(n)}\right)} \frac{\xi^{(n)}\left(\rho, x_{1}\right)}{\sum_{v \sim \rho} \xi^{(n)}(\rho, v)}=\frac{\xi^{(n)}\left(\rho, x_{1}\right)}{\operatorname{deg}\left(\rho^{(n)}\right)} \underset{n \rightarrow \infty}{\longrightarrow} 0 .
\end{aligned}
$$

In both cases

$$
\frac{\pi\left(\rho^{(n)}\right)}{\operatorname{deg}\left(\rho^{(n)}\right)} \hat{P}_{\omega(n)}\left(X_{-m}=x_{-m}, \ldots, X_{m}=x_{m}\right) \underset{n \rightarrow \infty}{\longrightarrow} 0=\frac{\pi(\rho)}{\operatorname{deg}(\rho)} \hat{P}_{\omega}\left(X_{-m}=x_{-m}, \ldots, X_{m}=x_{m}\right) .
$$

Analogously, we obtain the same convergence result, if there exists a $x_{-(k-1)}$ along the path in negative time with $\sum_{v \sim x_{-}(k-1)} \xi\left(x_{-(k-1)}, v\right)=0$, which implies the continuity of the last two factors integrated in (3.33).

Moreover, the first $m$ generations of the trees $T^{(n)}$ and $T$, rooted at $\rho^{(n)}$ and $\rho$, respectively, are equal for $n$ large enough. This implies $\left\{\left|T_{\mid B_{m}\left(\rho^{(n)}\right)}^{(n)}\right| \leq M\right\}=\left\{\left|T_{\mid B_{m}(\rho)}\right| \leq\right.$ $M\}$ for $n$ big enough. Thus, the function $\mathbb{1}_{\left\{|T|_{B_{m}(\rho)} \mid \leq M\right\}}$ is continuous in $\omega=(T, \rho, \xi)$, which completes the proof of the continuity of the functions integrated in (3.33). 
The speed of random walk on Galton-Watson trees with vanishing conductances

Using that also $\gamma_{\varepsilon} \rightarrow \gamma_{0}$, we obtain that

$$
\begin{aligned}
& \lim _{\varepsilon \rightarrow 0} \int f\left(\omega,\left(x_{n}\right)_{n \in \mathbb{Z}}\right) \mathbb{1}_{\left\{|T|_{B_{m}(\rho)} \mid \leq M\right\}} \mathrm{d} \hat{\mathbb{P}}_{\varepsilon} \\
& =\sum_{x_{-m}, \ldots, x_{m}} \int g\left(\omega,\left(x_{-m}, \ldots, x_{m}\right)\right) \mathbb{1}_{\left\{\left.T\right|_{B_{m}(\rho)} \leq M\right\}} \frac{\pi(\rho)}{\gamma_{0} \operatorname{deg}(\rho)} \hat{P}_{\omega}\left(X_{-m}=x_{-m}, \ldots, X_{m}=x_{m}\right) \mathrm{dP}_{0}^{\text {aug }} \\
& =\int f\left(\omega,\left(x_{n}\right)_{n \in \mathbb{Z}}\right) \mathbb{1}_{\left\{|T|_{B_{m}(\rho)} \mid \leq M\right\}} \mathrm{d} \hat{\mathbb{P}}_{0} .
\end{aligned}
$$

This computation shows that the first summand in (3.32) converges to zero for $\varepsilon \rightarrow 0$. Let us now consider the second one. We have

$$
\left|\int f \mathbb{1}_{\left\{\left|T_{\mid B_{m}(\rho)}\right|>M\right\}} \mathrm{d} \hat{\mathbb{P}}_{\varepsilon}\right| \leq\|f\|_{\infty} \mathrm{P}_{\varepsilon}^{\operatorname{aug}}\left(\left|T_{\mid B_{m}(\rho)}\right|>M\right) .
$$

The last probability does not depend on $\varepsilon$, and since $T$ is locally finite, we can bound this term by $\delta_{M}$ independent of $\varepsilon$ with $\delta_{M} \rightarrow 0$ for $M \rightarrow \infty$. For the third summand of (3.32) we obtain an analogous upper bound, which yields the weak convergence of $\hat{\mathbb{P}}_{\varepsilon}$ on $\mathcal{G} \otimes \mathcal{F}_{m}$.

To show the weak convergence on $\mathcal{G} \otimes \mathcal{F}$ we follow the arguments of [9, Theorem 2.2]. To apply the Portmanteau Theorem, note that $\mathcal{G} \otimes \mathcal{F}$ is the Borel- $\sigma$-algebra on $\Omega \times \mathbb{T}^{\mathbb{Z}}$. The sets $\left\{x \in \mathbb{T}^{\mathbb{Z}}: x_{-m}=v_{m}, \ldots, x_{m}=v_{m}\right\}$ with $v_{-m}, \ldots, v_{m} \in \mathbb{T}, m \in \mathbb{N}$ form a countable basis for the topology on $\mathbb{T}^{\mathbb{Z}}$, which is contained in $\bigcup_{m} \mathcal{F}_{m}$.

Let $G=G_{1} \times G_{2} \in \mathcal{G} \otimes \mathcal{F}$ be an open set. Hence, $G_{2}$ is open and since $\bigcup_{m} \mathcal{F}_{m}$ contains a countable basis of the topology, we can write $G_{2}=\bigcup_{i=1}^{\infty} A_{i}$ for some $A_{i} \in \bigcup_{m} \mathcal{F}_{m}$. By continuity of probability measures we have

$$
\hat{\mathbb{P}}_{0}\left(G_{1} \times \bigcup_{i=1}^{N} A_{i}\right) \underset{N \rightarrow \infty}{\longrightarrow} \hat{\mathbb{P}}_{0}\left(G_{1} \times G_{2}\right) .
$$

Given an arbitrary $\delta>0$ we can choose $r \in \mathbb{N}$, such that

$$
\hat{\mathbb{P}}_{0}\left(G_{1} \times \bigcup_{i=1}^{r} A_{i}\right)>\hat{\mathbb{P}}_{0}\left(G_{1} \times G_{2}\right)-\delta .
$$

We obtain

$$
\begin{aligned}
\liminf _{\varepsilon \rightarrow 0} \hat{\mathbb{P}}_{\varepsilon}\left(G_{1} \times G_{2}\right) & \geq \liminf _{\varepsilon \rightarrow 0} \hat{\mathbb{P}}_{\varepsilon}\left(G_{1} \times \bigcup_{i=1}^{r} A_{i}\right) \\
& \geq \hat{\mathbb{P}}_{0}\left(G_{1} \times \bigcup_{i=1}^{r} A_{i}\right) \\
& >\hat{\mathbb{P}}_{0}\left(G_{1} \times G_{2}\right)-\delta
\end{aligned}
$$

where the second inequality follows from the weak convergence of $\hat{\mathbb{P}}_{\varepsilon}$ on $\mathcal{G} \otimes \mathcal{F}_{m}$ and the Portmanteau Theorem and (3.35) was used for the last inequality. Since $\delta>0$ was arbitrary we have

$$
\liminf _{\varepsilon \rightarrow 0} \hat{\mathbb{P}}_{\varepsilon}(G) \geq \hat{\mathbb{P}}_{0}(G)
$$

for all open sets $G \in \mathcal{G} \otimes \mathcal{F}$, that is, $\hat{\mathbb{P}}_{\varepsilon}$ converges weakly to $\hat{\mathbb{P}}_{0}$. 
The speed of random walk on Galton-Watson trees with vanishing conductances

\section{References}

[1] Elie Aïdékon, Speed of the biased random walk on a Galton-Watson tree, Probab. Theory and Related Fields 159 (2014), no. 3-4, 597-617. MR-3230003

[2] Krishna B. Athreya and Peter E. Ney, Branching processes, Springer-Verlag, New YorkHeidelberg, 1972, Die Grundlehren der mathematischen Wissenschaften, Band 196. MR0373040

[3] Gérard Ben Arous and Alexander Fribergh, Biased random walks on random graphs, Probability and statistical physics in St. Petersburg 91 (2016), 99-153. MR-3526827

[4] Gérard Ben Arous, Alexander Fribergh, Nina Gantert, and Alan Hammond, Biased random walks on Galton-Watson trees with leaves, Ann. Probab. 40 (2012), no. 1, 280-338. MR2917774

[5] Gérard Ben Arous, Alexander Fribergh, and Vladas Sidoravicius, Lyons-Pemantle-Peres monotonicity problem for high biases, Communications on Pure and Applied Mathematics 67 (2014), no. 4, 519-530. MR-3168120

[6] Gérard Ben Arous, Yueyun Hu, Stefano Olla, and Ofer Zeitouni, Einstein relation for biased random walk on Galton-Watson trees, Annales de l'IHP Probabilités et statistiques, vol. 49, 2013, pp. 698-721. MR-3112431

[7] Noam Berger, Nina Gantert, and Jan Nagel, The speed of biased random walk among random conductances, Annales de l'Institut Henri Poincaré, Probabilités et Statistiques 55 (2019), no. 2, 862-881. MR-3949956

[8] Noam Berger, Nina Gantert, and Yuval Peres, The speed of biased random walk on percolation clusters, Probab. Theory Related Fields 126 (2003), no. 2, 221-242. MR-1990055

[9] Patrick Billingsley, Convergence of probability measures, John Wiley \& Sons, 2013. MR0233396

[10] Adam Bowditch, Escape regimes of biased random walks on Galton-Watson trees, Probability theory and related fields 170 (2018), no. 3, 685-768. MR-3773798

[11] Adam Bowditch and Yuki Tokushige, Differentiability of the speed of biased random walks on Galton-Watson trees, ALEA 17 (2020), 609-642. MR-4130577

[12] Irene Crimaldi and Luca Pratelli, Convergence results for conditional expectations, Bernoulli 11 (2005), no. 4, 737-745. MR-2158258

[13] David A Croydon, Alexander Fribergh, and Takashi Kumagai, Biased random walk on critical Galton-Watson trees conditioned to survive, Probability Theory and Related Fields 157 (2013), no. 1-2, 453-507. MR-3101853

[14] Peter G Doyle and J Laurie Snell, Random walks and electric networks, vol. 22, American Mathematical Soc., 1984. MR-0920811

[15] Alessandra Faggionato, Nina Gantert, and Michele Salvi, The velocity of 1d Mott variablerange hopping with external field, Annales de l'Institut Henri Poincaré, Probabilités et Statistiques 54 (2018), no. 3, 1165-1203. MR-3825879

[16] Alessandra Faggionato and Michele Salvi, Regularity of biased 1d random walks in random environment, ALEA 16 (2019), 1213-1248. MR-4030535

[17] Alexander Fribergh and Alan Hammond, Phase transition for the speed of the biased random walk on the supercritical percolation cluster, Communications on Pure and Applied Mathematics 67 (2014), no. 2, 173-245. MR-3149843

[18] Nina Gantert, Xiaoqin Guo, and Jan Nagel, Einstein relation and steady states for the random conductance model, The Annals of Probability 45 (2017), no. 4, 2533-2567. MR-3693969

[19] Nina Gantert, Sebastian Müller, Serguei Popov, and Marina Vachkovskaia, Random walks on Galton-Watson trees with random conductances, Stochastic Processes and their Applications 122 (2012), no. 4, 1652-1671. MR-2914767

[20] Remco van der Hofstad, Tim Hulshof, and Jan Nagel, Random walk on barely supercritical branching random walk, Probability Theory and Related Fields 177 (2020), no. 1, 1-53. MR-4095011 
The speed of random walk on Galton-Watson trees with vanishing conductances

[21] Jean-François Le Gall, Random trees and applications, Probability surveys 2 (2005), 245-311. MR-2203728

[22] Russell Lyons, Random walks, capacity and percolation on trees, Ann. Probab. 20 (1992), no. 4, 2043-2088. MR-1188053

[23] Russell Lyons, Robin Pemantle, and Yuval Peres, Ergodic theory on Galton-Watson trees: speed of random walk and dimension of harmonic measure, Ergodic Theory Dynam. Systems 15 (1995), no. 3, 593-619. MR-1336708

[24] Russell Lyons, Robin Pemantle, and Yuval Peres, Biased random walks on Galton-Watson trees, Probab. Theory Related Fields 106 (1996), no. 2, 249-264. MR-1410689

[25] Russell Lyons and Yuval Peres, Probability on trees and networks, Cambridge Series in Statistical and Probabilistic Mathematics, vol. 42, Cambridge University Press, New York, 2016. MR-3616205

[26] A. Pakes, Some limit theorems for the total progeny of a branching process, Advances in Applied Probability 3 (1971), no. 1, 176-192. MR-0283892

[27] A. W. Roberts and D. E. Varberg, Convex functions, Academic Press, 1973. MR-0442824

Acknowledgments. We thank two anonymous referees for helpful comments on an earlier version of this paper. 


\section{Electronic Journal of Probability Electronic Communications in Probability}

\section{Advantages of publishing in EJP-ECP}

- Very high standards

- Free for authors, free for readers

- Quick publication (no backlog)

- Secure publication $\left(\mathrm{LOCKSS}^{1}\right)$

- Easy interface (EJMS²)

\section{Economical model of EJP-ECP}

- Non profit, sponsored by $\mathrm{IMS}^{3}, \mathrm{BS}^{4}$, ProjectEuclid ${ }^{5}$

- Purely electronic

\section{Help keep the journal free and vigorous}

- Donate to the IMS open access fund ${ }^{6}$ (click here to donate!)

- Submit your best articles to EJP-ECP

- Choose EJP-ECP over for-profit journals

\footnotetext{
${ }^{1}$ LOCKSS: Lots of Copies Keep Stuff Safe http://www. lockss.org/

${ }^{2}$ EJMS: Electronic Journal Management System http://www.vtex.lt/en/ejms.html

${ }^{3}$ IMS: Institute of Mathematical Statistics http://www.imstat.org/

${ }^{4}$ BS: Bernoulli Society http://www. bernoulli-society.org/

${ }^{5}$ Project Euclid: https://projecteuclid.org/

${ }^{6}$ IMS Open Access Fund: http://www.imstat.org/publications/open.htm
} 